\title{
Episodes of Fall Asleep During Day Time in an Elder Woman with Vascular Dementia: Impact on Cerebral Ischeamic Tolerance and Utility of ECG Holter Monitoring
}

\author{
Michele M. Ciulla ${ }^{1,2, *}$, Giulia Acquistapace ${ }^{1}$, Roberta Paliotti ${ }^{1}$, Chiara Benfenati ${ }^{1}$ \\ and Fabio Magrini ${ }^{1}$
}

\begin{abstract}
${ }^{1}$ Department of Respiratory and Cardiovascular Disease, Laboratory of Clinical Informatics and Cardiovascular Imaging, University of Milan, Milan, Italy; Fondazione IRCCS Ca' Granda, Ospedale MAggiore Policlinico, Milan, Italy; ${ }^{2}$ Istituti Clinici di Perfezionamento, Poliambulatori, ECG Holter Monitoring Service
\end{abstract}

\begin{abstract}
Here we report the case of an 86-year-old woman with advanced dementia addressed to our service for routinary ECG Holter Monitoring (EHM) for bradycardia in AV block type I. Several day-time episodes of fall-asleep while sitting had been previously reported by the nurse and generally attributed to the dementia itself, without taking into consideration the hypothesis of an AV block. The EHM reading reported several and often subsequent pauses (561), many of them critical, the longest lasting 15,9 s with no changes in clinical condition of the patient. The results of the EHM were reported to the physicians in charge for the patient and subsequently the woman was referred to the arrhythmology unit for pace-maker device implantation. Generalizing our experience, we suggest that advanced dementia, often associated with episodes of fall-asleep, could mask a conduction disturbance causing critical pauses with syncope; therefore we suggest screening those patients for possible arrhythmic disorders. Finally, we remark that in our patient the pauses weren't associated with a worsening of the patient as seen in the follow-up, and this fact supports the hypothesis that vascular dementia could increase cerebral ischaemic tolerance.
\end{abstract}

Keywords: ECG Holter Monitoring, advanced dementia, total AV block, long ECG pauses, cerebral ischaemic preconditioning and tolerance.

\section{INTRODUCTION}

Several episodes of fall-asleep during daytime are known to occur in elder patients with cognitive disease related to advanced dementia (AdD) on the basis of a chronic cerebral vascular disease [1]. This increased tendency to fall-asleep during day-time and the associated increase to wakefulness during the night suggest that in AdD an altered sleep/wake rhythm occurs. This alteration is probably related to the cognitive decline [5] and this may lead physicians to avoid further investigation on other possible causes of this evidence. Here we report the case of an AdD 86-year-old woman addressed as out-patient to our service for routinary ECG Holter Monitoring (EHM) for bradycardia. The patient was housed in a retirement home, the only information known about the patient were extremely general: she was affected by arterial hypertension and vascular dementia confirmed by neuroimaging (without information about age of onset or therapy). During the previous months the nurse had reported several episodes of fall-asleep during day-time in sitting position (wheelchair). At basal ECG a AV block type I was identified. At the moment of the request, no echocardiographic examinations were available.

*Address correspondence to this author at the Department of Respiratory and Cardiovascular Disease, University of Milan, via F.Sforza, 35 - Milan, 20122 Italy; Tel: +39.0255033592; Fax: +39.0250320480;

E-mail: michele.ciulla@unimi.it
The EHM reading reported sinus rhythm with the mean frequency of $62 \mathrm{bpm}$ (range $36-90 \mathrm{bpm}$ ), AV block type I (PQ $0.22 \mathrm{sec}$ ), frequent episodes of AV block type II type 1 (2:1) and type 2, and episodes of total AV block with occasional escape ventricular beats at $36 \mathrm{bpm}$; several and often subsequent pauses (561) (Fig. 1), many of whom were critical (> $200 \mathrm{msec}$ ), the longest lasting $1590 \mathrm{msec}$ (Fig. 2). Concurrently with these pauses, a note in the daily diary reported by the nurse described a short-lasting periods of falling asleep, while the patient was in the sitting position. Frequent ectopic supraventricular beats were also reported (2763), associated with several episodes of supraventricular tachycardia. No significant alterations of ST tract and ventricular repolarization were found.

The results of the EHM were reported to the physicians in charge for the patient and subsequently the woman was referred to the arrhythmology unit for pace-maker device implantation, according to the guidelines [3]. During the first 6 month of follow-up, no major events occurred and day time episodes of fall asleep were generally reduced.

\section{DISCUSSION}

Syncope is defined as a self-limited loss of consciousness, usually combined with falling due to the inability to maintain postural tone. The underlying mechanism is a transient global cerebral hypoperfusion. The aetiology essen- 


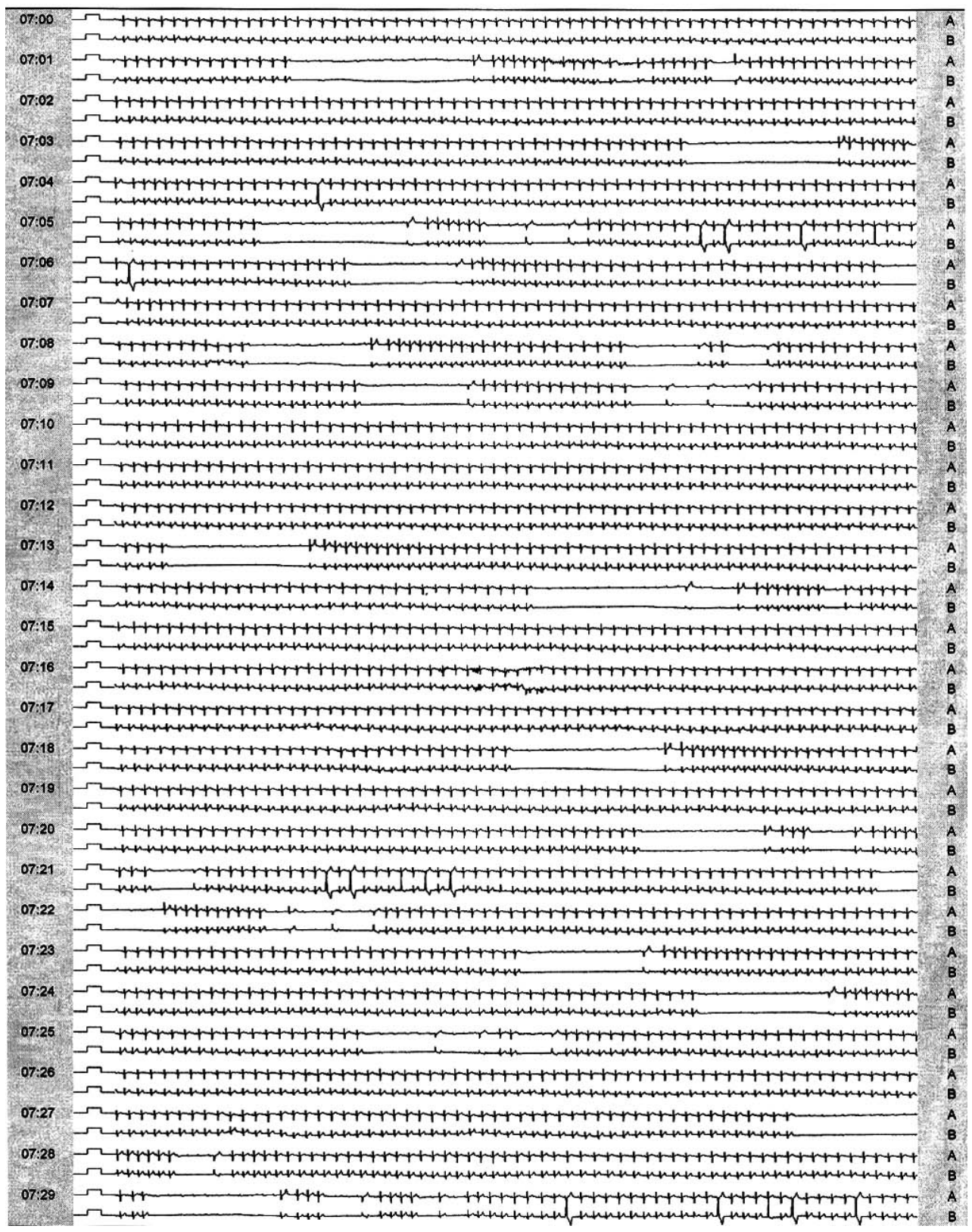

Fig. (1). A sample of 29 minutes (from 07:00 am to 07:29 am) ECG Holter monitoring tracing showing several pauses of more than 2 sec $(n=31)$.

tially includes cardiac disorders (structured heart disease or arrhythmias), neurally-mediated reflex syndromes, orthostatic hypotension and carotid sinus syndrome. History and physical examination will lead to the diagnosis in up to $50 \%$ of cases [2]. In patients with arrhythmic syncope, a poor prognosis can be expected if syncope is misdiagnosed, particularly when arrhythmias occur in a patient with advanced heart disease [3]. The most important step is to differentiate patients with heart disease from others, since the mortality of these patients is doubled [3]. EHM is one of the most useful techniques to approach this population: it is affordable and valuable for the investigation of heart rate and rhythm over a long period of time [4]. According to the flow chart for diagnosis of syncope in the elderly [2], episodes described as short-lasting periods of falling asleep could be interpreted as cardiac syncope. In these patients, the onset of syncope is rapid, usually without premonitory symptoms, the recovery is spontaneous with almost immediate restoration of appropriate behavior and orientation [6] and no resuscitation is needed. A typical syncopical episode is shorter than $20 \mathrm{~s}$ and in the rare cases in which syncope duration is longer, the differentiation between syncope and other causes of loss of consciousness can be difficult. Within 5 to $15 \mathrm{~s}$ after cerebral hypoperfusion loss of consciousness, paleness and loss of muscle tone with subsequent falling occur. After $>15 \mathrm{~s}$ of hypoperfusion, there may be jerking movements including urinary or faecal incontinence, which has to be differentiated mainly from epilepsy. Some disorders in which loss of consciousness does not result from sudden transient global cerebral hypoperfusion are misdiagnosed as syncope [2]. 


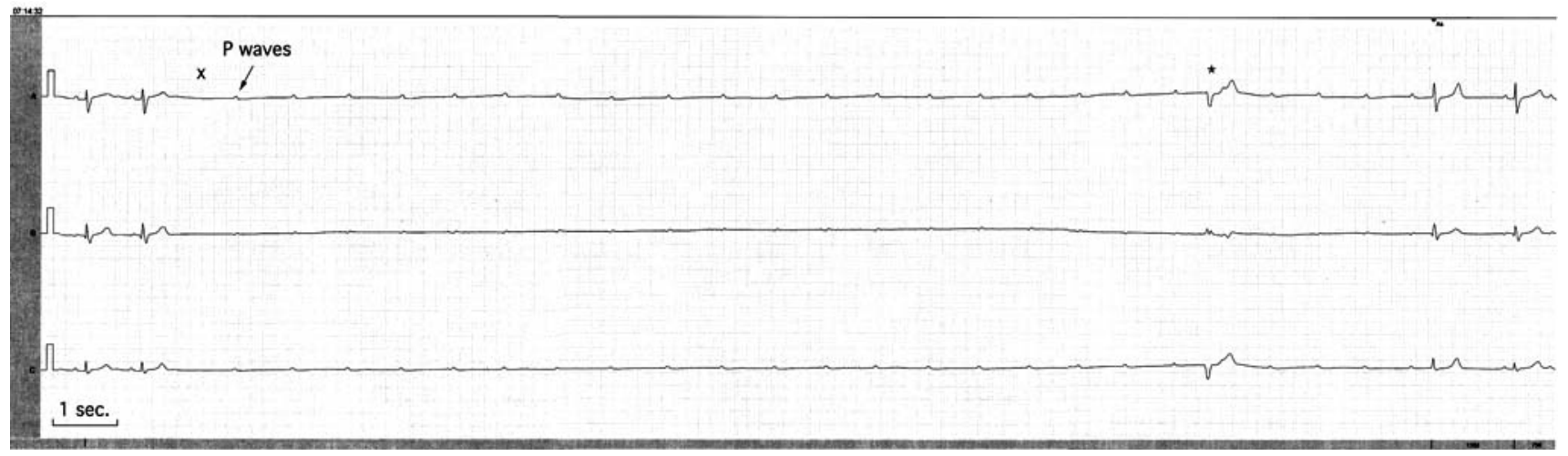

Fig. (2). Original ECG Holter monitoring tracing showing the longest pause lasting 15,9 sec, caused by a total AV block during day time. Concurrently with these pauses, a note in the daily diary reported by the nurse describes a short-lasting "period of falling asleep", while the patient was in the sitting position. Unconducted $\mathrm{P}$ waves are clearly evident at frequency of $75 \mathrm{bpm}$. Before the end of the pause, a ventricular escape beat is evident.

In our case, the syncopes duration were borderline (about $15 \mathrm{sec}$ ), very frequent, often subsequent and the sitting position on a wheelchair prevented the patient from falling down. Furthermore, the coexistence of an $\mathrm{AdD}$, often associated with fall asleep, masked the association with the conduction disturbance. Therefore, the EHM was requested for the bradycardia in pre-existing AV block type I, in order to screen for possible AV blocks that are very common in elderlies.

The most interesting feature is the ischaemic threshold of this patient. We can hypothesize a correlation between dementia and ischaemic tolerance from a vascular point of view, since in our patient the pauses weren't associated to a clinical worsening of the patient, that was confirmed by NMR imaging done in the follow up and compared with the previous one.

Arrhythmic syncope can be easily prevented with antiarrhythmic drugs or, more frequently, with non-medical treatments such as pacemaker or defibrillator implantation [3]. In our case, the patient underwent a pace-maker implantation.

\section{CONCLUSION}

Generalizing our reported experience, when an old woman falls asleep several times a day in the sitting position we suggest to screen for possible arrhythmic disorder that are very common causes of paroxysmal complete A-V block, generally related to an infra- or intrahisian A-V block characteristic of the Adams-Stokes syndrome [3], and this irrespective of a coexistent AdD. This experience suggests to consider EHM in elderly patients with episodes of fall-asleep in the context of dementia. The sitting position on a wheelchair could be misleading since it prevents patients from falling down. Furthermore, we cannot exclude that in a pa- tient with an history of chronic cerebrovascular impairment responsible for dementia, cerebral ischaemic tolerance is different (probably increased) [7]. It is well known that organisms have evolved mechanisms to protect against tissue damage and to compensate in the event of injury. One of the pathophysiological mechanisms supposed is preconditioning, by means of which an organ becomes resistant or tolerant to a noxious stimuli when it has been given before under the threshold of damage [8]. Nevertheless, we cannot exclude that the decrease of cardiac load, determined by a preexisting bradycardia in AV block, could be the primary cause of this process [9].

\section{REFERENCES}

[1] Bliwise, DL. Sleep in normal aging and dementia. Sleep 1993; 16: 40-81.

[2] Wöhrle J, Kochs M. Syncope in the elderly. Z Gerontol Geriatr 2003; 36: 2-9.

[3] Brambilla-Perrot B. The management of arrhythmic sincope. Minerva Medica 2009; 100: 195-211.

[4] Southhall DP, Johnston F, Shinebourne EA, Johnston PGB. 24hour electocardiographic study of heart rate and rhythm patterns in population of healthy children. Br Heart 1981; 45: 281-91.

[5] Bonanni E, Maestri M, Tognoni G, Fabbrini M, Nucciarone B, Manca ML, Gori S, Iudice A, Murri L. Daytime sleepiness in mild and moderate Alzheimer's disease and its relationship with cognitive impairment. J Sleep Res 2005; 14: 311-7.

[6] Brignole M, Alboni P, Benditt D, et al. Guidelines on management (diagnosis and treatment) of syncope. Eur Heart J 2001; 22: 12563067.

[7] Gidday JM. Cerebral preconditioning and ischaemic tolerance. Nat Rev Neurosci 2006; 7: 437-48.

[8] Dirnagl U, Becker K, Meisel A. Preconditioning and tolerance against cerebral ischaemia: from experimental strategies to clinical use. Lancet Neurol 2009; 8: 398-412.

[9] Weimer LH, Zadeh P. Neurological aspect of syncope and orthostatic intolerance. Med Clin North Am 2009; 93: 427-49. 